\title{
A notion of homotopy for the effective topos
}

\author{
JAAP VAN OOSTEN \\ Department of Mathematics, Utrecht University, P.O. Box 80.010, 3508 TA Utrecht, the Netherlands \\ E-mail: J.vanOosten@uu.nl
}

Received 1 March 2010; revised 20 January 2014

We define a notion of homotopy in the effective topos.

\section{Introduction}

This paper deals with a notion of 'homotopy' one can define in the effective topos of Hyland (1982).

In recent years, there has been an upsurge of interest in connections between abstract homotopy theories and type theory: see Gambino and Garner (2008), Warren (2008), Awodey and Warren (2009), Voevodsky (2010), Van den Berg and Garner (2011), Kapulkin et al. (2012) and Van den Berg and Garner (2012). A prominent area of focus is that of (closed) model categories, first defined by Quillen (1967); for modern expositions see Hovey (1999), Dwyer and Spalinski (1995) and Hirschhorn (2003). A closed model structure on a category defines a notion of homotopy, and this is used in order to model the identity types of Martin-Löf's type theory. For a comprehensive recent account, see Univalent (2013).

In this paper, the focus is on a topos that is often considered an oddity in the world of toposes and chiefly useful for applications in logic. However, we show that it is possible to think of the objects of this topos as spaces and understand the effective topos in geometric (topological) terms.

It is shown that a sensible notion of 'path object', for every object of the topos, arises out of the so-called 'discrete reflection'. In the effective topos, the full subcategory of discrete objects has been thoroughly studied (see, e.g. Hyland et al. (1990)). I remind the reader that an object of the effective topos is called 'discrete' if it is a quotient of a subobject of the natural numbers object. There are several equivalent characterizations of the discrete objects, among which:

An object $X$ is discrete if and only if the canonical map from $X$ to the exponential $X^{\mathcal{P}(N)}$ is an isomorphism, where $\mathcal{P}(N)$ denotes the power object of the natural numbers.

Easy proofs of this and related facts may be found in Van Oosten (2008). The fact above means that the discrete objects are defined by an orthogonality property, and hence enjoy good completeness properties relative to the effective topos. In particular, the inclusion of the discrete objects as full subcategory of the effective topos has a left adjoint, which is called the 'discrete reflection'. 
Part of the significance of the present paper is to provide a new and very concrete, intuitive meaning to the word 'discrete'. Indeed, my notion of 'path' will be such that:

1. An object $X$ is discrete if and only if there are no nontrivial (= nonconstant) paths in $X$.

2. The discrete reflection of $X$ is (internally) the set of path components of $X$.

This should lead, hopefully, to a study of 'topological' properties of non-discrete objects. For, the non-discrete part of the effective topos has not been studied nearly as extensively as the discrete part (for example, the following question has, to my knowledge, not been answered: which objects $Y$ have the property that $X \rightarrow X^{Y}$ is an isomorphism, for every discrete object $X$ ?).

Note, by the way, that the properties of 'paths' above imply that there is no connection to the real line. Indeed, in the effective topos the object of real numbers is discrete. This is nothing unusual in the study of abstract homotopy theory: after all, in the topos of simplicial sets the standard interval has nothing to do with the real numbers object of the topos (whose geometric realization is a discrete space). We are studying a notion which is similar in spirit to the homotopy in a closed model category.

One may doubt whether the terminology 'discrete' for the objects of the effective topos described above, was well-chosen. However, I do not feel that this paper is the place to quarrel with established language.

We obtain sensible interpretations of standard notions from topology. For example, an object is path connected if and only if its discrete reflection is isomorphic to the terminal object; an object is simply connected if it is path connected and its fundamental group is trivial. We shall see that these notions do not coincide: we have 'circles' whose fundamental group is isomorphic to $\mathbb{Z}$. We also define 'spheres'.

In the final section of this paper, we define Hurewicz fibrations, strong deformation retracts and homotopy equivalences in a natural way. We show that every arrow in the effective topos has a factorization as a strong deformation retract followed by a Hurewicz fibration. The resulting structure should satisfy Baues' axioms for a fibration category (there is still an embarrassing open question) and Van den Berg and Garner's axioms for a path object category.

Many questions remain for future work. Is there a model structure on the effective topos with Hurewicz fibrations and homotopy equivalences as fibrations and weak equivalences, respectively? What does the homotopy category of the effective topos look like? It seems obvious that the construction of this paper can be performed in arbitrary 'realizability toposes'. Is there a generalization to toposes coming from an arbitrary tripos?

\subsection{Preliminaries}

For the sake of self-containedness, I give the definition of the effective topos as a category. From now on, it will be denoted by $\mathcal{E} f f$. You are referred to Hyland (1982) and Van Oosten (2008) for further information. 
The category $\mathcal{E} f f$ is built on the notion of a computable function on the natural numbers.

An object is a pair $(X, \sim)$ where $X$ is a set and $\sim$ is a function from $X \times X$ to the set $P(\mathbb{N})$ of subsets of $\mathbb{N}$, usually written as $x, y \mapsto[x \sim y]$. This function $\sim$ has to satisfy the requirement that there exist computable functions $s$ (symmetry) and $t$ (transitivity), such that the following hold:

- if $a \in[x \sim y]$ then $\mathrm{s}(a) \in[y \sim x]$,

- if $a \in[x \sim y]$ and $b \in[y \sim z]$ then $\mathrm{t}(a, b) \in[x \sim z]$.

The set $[x \sim y]$ is called the equality of $x$ and $y$.

Given two objects $(X, \sim)$ and $(Y, \approx)$, a morphism $(X, \sim) \rightarrow(Y, \approx)$ is represented by a function $F: X \times Y \rightarrow P(\mathbb{N})$ for which there exist computable functions st $_{X}$, st st $_{Y}$ (strictness), rel $_{X}$, rel $_{Y}$ (relationality), tl (totality) and sv (single-valuedness), satisfying:

- if $a \in F(x, y)$ then $\operatorname{st}_{X}(a) \in[x \sim x]$ and $\operatorname{st}_{Y}(a) \in[y \approx y]$,

- if $a \in F(x, y), b \in\left[x^{\prime} \sim x\right]$ and $c \in\left[y \approx y^{\prime}\right]$ then $\operatorname{rel}_{X}(b, a) \in F\left(x^{\prime}, y\right)$ and $\operatorname{rel}_{Y}(a, c) \in$ $F\left(x, y^{\prime}\right)$,

- if $a \in[x \sim x]$ then $\operatorname{tl}(a) \in \bigcup_{y \in Y} F(x, y)$,

- if $a \in F(x, y)$ and $b \in F\left(x, y^{\prime}\right)$ then $\operatorname{sv}(a, b) \in\left[y \approx y^{\prime}\right]$.

Two such functions $F, G$ determine the same morphism $(X, \sim) \rightarrow(Y, \approx)$ if there is a computable function $\phi$ such that whenever $a \in F(x, y), \phi(a) \in G(x, y)$ (this is an equivalence relation on functions which represent a morphism).

We shall write $\left\langle a_{1}, \ldots, a_{n}\right\rangle$ for the natural number which codes the finite tuple of natural numbers $\left(a_{1}, \ldots, a_{n}\right)$ in some coding for which all the operations one wishes to perform are given by computable functions: e.g. determining the length of a sequence coded by $x$, computing the code of the sequence which is the concatenation of the sequences coded by $x$ and $y$, determining the $i$ th element of the sequence coded by $x$, etc.

For an object $(X, \sim)$ and $x \in X$ we shall often write $E(x)$ for $[x \sim x] . E(x)$ is called the existence of $x$.

Example 1. Suppose $(X, \sim)$ and $(Y, \approx)$ are objects of $\mathcal{E f f}$ and $f: X \rightarrow Y$ is a function such that there exist computable functions $\phi$ and $\psi$ satisfying:

- whenever $a \in E(x), \phi(a) \in E(f(x))$,

- whenever $a \in\left[x \sim x^{\prime}\right], \psi(a) \in\left[f(x) \approx f\left(x^{\prime}\right)\right]$.

Then the following function $F$ represents a morphism $(X, \sim) \rightarrow(Y, \approx)$ :

$$
F(x, y)=\bigcup_{x^{\prime} \in X}\left\{\langle a, b\rangle \mid a \in\left[x \sim x^{\prime}\right], b \in\left[f\left(x^{\prime}\right) \approx y\right]\right\} .
$$

Let us say that this morphism is induced by the function $f$.

Example 2. If $(X, \sim)$ is an object of $\mathcal{E} f f$, then a subobject of $(X, \sim)$ is determined by a function $F: X \rightarrow P(\mathbb{N})$ for which there exist computable functions $\phi$ and $\psi$ satisfying

- if $a \in F(x)$ then $\phi(a) \in E(x)$,

- if $a \in F(x)$ and $b \in\left[x \sim x^{\prime}\right]$ then $\psi(a, b) \in F\left(x^{\prime}\right)$. 
The subobject determined by $F$ is then represented by the object $\left(X, \sim^{\prime}\right)$ where

$$
\left[x \sim^{\prime} y\right]=\{\langle a, b\rangle \mid a \in F(x), b \in[x \sim y]\} .
$$

Example 3. An assembly is an object $(X, \sim)$ which is such that $[x \sim y]=\varnothing$ if $x \neq y$. Hence, an assembly is given by a map $E: X \rightarrow P(\mathbb{N})$. The assemblies are (up to isomorphism) the $\neg \neg$-separated objects of $\mathcal{E} f f$. An assembly is called canonically projective if $E(x)$ is a singleton for each $x$. These are (up to isomorphism) the projective objects of $\mathcal{E} f f$.

Every morphism into an assembly is induced by a unique function on the level of sets, as in Example 1.

\section{Intervals and paths}

Since, in $\mathcal{E} f f$, the object of real numbers is discrete, it will come as no surprise that the 'intervals' defined here have nothing to do with the unit interval $[0,1]$ of real numbers.

Definition 2.1. Let $A=\left\{\alpha_{0}, \alpha_{1}, \ldots\right\}$ and $B=\left\{\beta_{0}, \beta_{1}, \ldots\right\}$ be two disjoint countable sets. The generic interval of length $n$ is the object $(X, \sim)$ where $X=\left\{\alpha_{0}, \ldots, \alpha_{n}, \beta_{0}, \ldots, \beta_{n}\right\}$ and $\sim$ is given by:

$$
\begin{array}{ll}
{\left[\alpha_{i} \sim \alpha_{i}\right]=\{\langle i\rangle\}} & {\left[\alpha_{i} \sim \alpha_{j}\right]=\varnothing \text { if } i \neq j} \\
{\left[\beta_{i} \sim \beta_{i}\right]=\{\langle i+1\rangle\}} & {\left[\beta_{i} \sim \beta_{j}\right]=\varnothing \text { if } i \neq j} \\
{\left[\alpha_{i} \sim \beta_{i}\right]=\{\langle i, i+1\rangle\}} & {\left[\alpha_{i} \sim \beta_{j}\right]=\varnothing \text { if } i \neq j} \\
{\left[\beta_{i} \sim \alpha_{i}\right]=\{\langle i+1, i\rangle\}} & {\left[\beta_{i} \sim \alpha_{j}\right]=\varnothing \text { if } i \neq j}
\end{array}
$$

This object is denoted $I_{n}$.

I prefer to visualize the object $I_{n}$ in the following way:

$$
\begin{aligned}
& \alpha_{n} \frac{\langle n+1, n\rangle}{\langle n, n+1\rangle} \beta_{n} \\
& \alpha_{2} \frac{\langle 3,2\rangle}{\langle 2,3\rangle} \cdots \\
& \alpha_{1} \frac{\langle 2,1\rangle}{\langle 1,2\rangle} \beta_{1} \\
& \alpha_{0} \frac{\langle 1,0\rangle}{\langle 0,1\rangle} \beta_{0} \\
& \begin{array}{llllll}
0 & 1 & 2 & \cdots & n & n+1
\end{array}
\end{aligned}
$$

That is, nontrivial equalities are given by labelled horizontal lines; elements on the same vertical line have the same existence.

The following facts about $I_{n}$ are easily established and left to the reader.

\section{Proposition 2.2.}

1. Let $K_{n}$ be the canonically projective object $\left(\left\{\alpha_{0}, \ldots, \alpha_{n}, \beta_{0}, \ldots, \beta_{n}\right\}, E\right)$ with $E\left(\alpha_{i}\right)=\{i\}$ and $E\left(\beta_{i}\right)=\{i+1\}$. Let $[n+1]$ be the canonically projective object (finite cardinal) $(\{0, \ldots, n\}, E)$ with $E(i)=\{i\}$. Then there are morphisms $f, g:[n+1] \rightarrow K_{n}$, given by 
$f(i)=\alpha_{i}, g(i)=\beta_{i}$, such that there is a coequalizer diagram

$$
[n+1] \stackrel{f}{\underset{g}{\longrightarrow}} K_{n} \longrightarrow I_{n} .
$$

2. The object $I_{n}$ is $\neg \neg$-separated, and isomorphic to the assembly $\tilde{I}_{n}=(\{0, \ldots, n\}, E)$ with $E(i)=\{i, i+1\}$.

Fact (i) of Proposition 2.2 makes it easy to describe the exponentials $(X, \sim)^{I_{n}}$. For, we have an equalizer diagram

$$
(X, \sim)^{I_{n}} \longrightarrow(X, \sim)^{K_{n}} \longrightarrow(X, \sim)^{[n+1]}
$$

and hence, by the well-known formation of exponentials in the case that the exponent is canonically projective, we see that $(X, \sim)^{I_{n}}$ has as underlying set the set of functions from $\left\{\alpha_{0}, \ldots, \alpha_{n}, \beta_{0}, \ldots, \beta_{n}\right\}$ to $X$, where the existence of such a function $f$ is the set of coded pairs $\langle\sigma, \tau\rangle$ such that $\sigma$ is a coded tuple $\left\langle c_{0}, \ldots, c_{n+1}\right\rangle$ satisfying $c_{i} \in E\left(f\left(\alpha_{i}\right)\right)$ for $0 \leqslant i \leqslant n$ and $c_{i} \in E\left(f\left(\beta_{i-1}\right)\right)$ for $1 \leqslant i \leqslant n+1$; and $\tau$ is a coded tuple $\left\langle a_{0}, \ldots, a_{n}\right\rangle$ such that $a_{i} \in\left[f\left(\alpha_{i}\right) \sim f\left(\beta_{i}\right)\right]$ for $0 \leqslant i \leqslant n$. The equality between two such functions $f$ and $g$ is the set of coded tuples $v=\left\langle d_{0}, \ldots, d_{n+1}\right\rangle$ such that $d_{i} \in\left[f\left(\alpha_{i}\right) \sim g\left(\alpha_{i}\right)\right]$ for $0 \leqslant i \leqslant n$, and $d_{i} \in\left[f\left(\beta_{i-1}\right) \sim g\left(\beta_{i-1}\right)\right]$ for $1 \leqslant i \leqslant n+1$.

Definition 2.3. A morphism $I_{n} \stackrel{F}{\rightarrow} I_{m}$ is called order and endpoint preserving if the unique function $f:\{0, \ldots, n\} \rightarrow\{0, \ldots, m\}$ which induces the corresponding arrow $\tilde{I}_{n} \rightarrow \tilde{I}_{m}$ (via the isomorphism of 2.2 2) ), is order-preserving and satisfies $f(0)=0, f(n)=m$.

Proposition 2.4. Every order and endpoint preserving map $I_{n} \rightarrow I_{m}$ is surjective; hence such maps exist if and only if $n \geqslant m$.

Proof. Consider $f:\{0, \ldots, n\} \rightarrow\{0, \ldots, m\}$. If $i \in\{0, \ldots, m\}$ is not in the image of $f$ then $i \neq 0$ and $i \neq m$ (since $f$ is endpoint preserving) so there must be an element $j \in\{0, \ldots, n\}$ such that $f(j)<i$ and $f(j+1)>i$. But then, in $\tilde{I}_{m}, E(f(j)) \cap E(f(j+1))=\varnothing$ whereas in $\tilde{I}_{n},\langle j+1\rangle \in E(j) \cap E(j+1)$. So $f$ cannot induce a morphism in $\mathcal{E} f f$.

Definition 2.5. Let $(X, \sim)$ be an object of $\mathcal{E} f f$. The path object of $(X, \sim)$, denoted $\mathrm{P}(X, \sim)$, is defined as follows.

Its underlying set is the set of all pairs $(n, f)$ with $n \in \mathbb{N}$ and $f$ is a function from $\left\{\alpha_{0}, \beta_{0}, \ldots, \alpha_{n}, \beta_{n}\right\}$ to $X$.

Given two such pairs $(n, f)$ and $(m, g)$, the equality $[(n, f) \sim(m, g)]$ between them is the set of all coded triples $\langle a, s, b\rangle$ satisfying:

a. $a \in E(f)$ as element of $(X, \sim)^{I_{n}}$,

b. $b \in E(g)$ as element of $(X, \sim)^{I_{m}}$,

c. there is an order and endpoint preserving morphism $\sigma: I_{n} \rightarrow I_{m}$ such that $s \in[f \sim g \sigma]$ in the sense of $(X, \sim)^{I_{n}}$; or there is an order and endpoint preserving morphism $\sigma: I_{m} \rightarrow I_{n}$ such that $s \in[f \sigma \sim g]$ in the sense of $(X, \sim)^{I_{m}}$.

Remark. It may seem, since our intervals have variable length, that we are modelling a kind of 'Moore-path'. However, this is misleading: the nature of the equality relation on 
$\mathrm{P}(X, \sim)$ precludes that we have a function: $\mathrm{P}(X, \sim) \rightarrow N$ giving the length of a path (here, $N$ denotes the natural numbers object in the effective topos).

\section{Proposition 2.6.}

i. The construction of $\mathrm{P}(X, \sim)$ extends to a functor $\mathrm{P}: \mathcal{E} f f \rightarrow \mathcal{E} f f$ which preserves finite limits.

ii. The object $\mathrm{P}(X, \sim)$ comes equipped with well-defined maps:

$\mathrm{s}($ source $), \mathrm{t}$ (target): $\mathrm{P}(X, \sim) \rightarrow(X, \sim)$

c (trivial path $):(X, \sim) \rightarrow \mathrm{P}(X, \sim)$

* (composition of paths): $\mathrm{P}(X, \sim) \times_{(X, \sim)} \mathrm{P}(X, \sim) \rightarrow \mathrm{P}(X, \sim)$, where the domain is the pullback

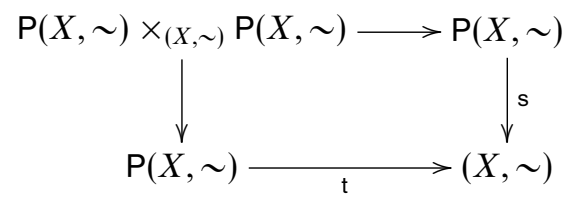

$\widetilde{(\cdot)}$ (converse path): $\mathrm{P}(X, \sim) \rightarrow \mathrm{P}(X, \sim)$.

With these data, $\mathrm{P}(X, \sim)$ is an internal category in $\mathcal{E} f f$ which has a contravariant involution which is the identity on objects.

Proof. I leave most of this to the reader. That $\mathrm{P}$ preserves products is a consequence of the equality relation we defined on $\mathrm{P}(X, \sim)$, which ensures that we 'can assume that two paths are of the same length, i.e. for $(n, f)$ representing an element of $\mathrm{P}(X, \sim)$ and $a \in E(n, f)$ we can, for each $m \geqslant n$, find a canonical element $\left(m, f^{\prime}\right)$ and an element of $\left[(n, f) \sim\left(m, f^{\prime}\right)\right]$.

For functions $f:\left\{\alpha_{0}, \beta_{0}, \ldots, \alpha_{n}, \beta_{n}\right\} \rightarrow X$ and $g:\left\{\alpha_{0}, \beta_{0}, \ldots, \alpha_{m}, \beta_{m}\right\} \rightarrow X$ let $f * g:$ $\left\{\alpha_{0}, \beta_{0}, \ldots, \alpha_{n+m}, \beta_{n+m}\right\} \rightarrow X$ be the function defined by

$$
\begin{aligned}
& f * g\left(\alpha_{i}\right)=\left\{\begin{array}{l}
f\left(\alpha_{i}\right) \quad i \leqslant n \\
g\left(\alpha_{i-n}\right) i>n
\end{array}\right. \\
& f * g\left(\beta_{i}\right)=\left\{\begin{array}{l}
f\left(\beta_{i}\right) \quad i<n \\
g\left(\beta_{i-n}\right) i \geqslant n
\end{array}\right.
\end{aligned}
$$

Then composition of paths is represented by the function which sends a triple $(n, f)$, $(m, g),(k, h)$ to the set of coded 4-tuples $\langle a, b, c, d\rangle$ such that $a \in E(f), b \in E(g), c \in$ $\left[f\left(\beta_{n}\right) \sim g\left(\alpha_{0}\right)\right]$ and $d \in[(k, h) \sim(n+m, f * g)]$. Here $E(f), E(g)$ refer to the existence of $(X, \sim)^{I_{n}},(X, \sim)^{I_{m}}$ respectively.

Composition is strictly associative. It is another consequence of the particular equality on $\mathrm{P}(X, \sim)$ that the trivial (constant) paths are strict identities for composition. 
For the following proposition, I find it convenient to visualize paths in $(X, \sim)$ in the way of the picture for $I_{n}$; so if $f:\left\{\alpha_{0}, \beta_{0}, \ldots, \alpha_{n}, \beta_{n}\right\} \rightarrow X$ represents such a path, $x_{i}=f\left(\alpha_{i}\right)$ and $y_{i}=f\left(\beta_{i}\right)$ and $\langle\sigma, \tau\rangle \in E(f)$ in the sense of $(X, \sim)^{I_{n}}$, so $\sigma=\left\langle c_{0}, \ldots, c_{n+1}\right\rangle, \tau=\left\langle a_{0}, \ldots, a_{n}\right\rangle$, we picture $f$ as

$$
\begin{aligned}
& x_{n} \frac{a_{n}}{y_{n}} \\
& x_{1} \frac{a_{1}}{a_{1}} \\
& x_{0} \frac{a_{0}}{a_{0}} \\
& \begin{array}{ccccccc}
c_{0} & c_{1} & c_{2} & \ldots & c_{n} & c_{n+1}
\end{array}
\end{aligned}
$$

Such a path proceeds (in $X$ ) by alternately taking a horizontal and a vertical step: a horizontal step involves an equality in $(X, \sim)$, a vertical step involves an element in the intersection of the existences.

Proposition 2.7. There is a morphism $\mathrm{P}(X, \sim) \times_{(X, \sim)} \mathrm{P}(X, \sim) \stackrel{L}{\rightarrow} \mathrm{PP}(X, \sim)$ such that $\mathrm{s}(L(f, g))=f * g$ and $\mathrm{t}(L(f, g))=g$.

Proof. We do this for the special case that $g$ is constant; the generalization to the statement in the proposition is straightforward and left to the reader.

So, we wish to show that there is $L: \mathrm{P}(X, \sim) \rightarrow \operatorname{PP}(X, \sim)$ such that $\mathrm{s} L=\mathrm{id}_{\mathrm{P}(X, \sim)}$ and $\mathrm{t} L=\mathrm{ct}$ :
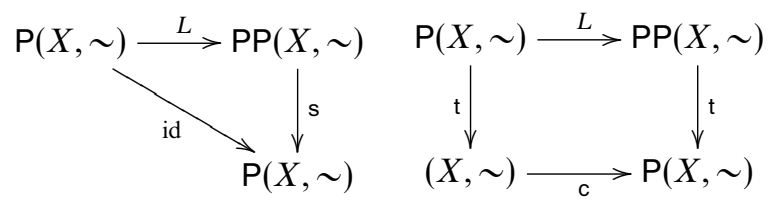

First we show that there is a map $L_{k}:(X, \sim)^{I_{k}} \rightarrow\left((X, \sim)^{I_{k}}\right)^{I_{k}}$ with these properties. It is induced by the function $\ell_{k}$ which we now describe:

Suppose $f$ represents an element of $(X, \sim)^{I_{k}}$ :

$$
x_{k} \frac{y_{k}}{a_{k}}
$$

$$
\begin{aligned}
& x_{1} \frac{a_{1}}{a_{1}} \\
& x_{0} \frac{y_{0}}{a_{0}} y_{0} \\
& \begin{array}{cccccc}
c_{0} & c_{1} & c_{2} & \ldots & c_{k} & c_{k+1}
\end{array}
\end{aligned}
$$


Then $\ell_{k}(f)$ is the path $\left(f_{0}, p_{0}, \ldots, f_{k}, p_{k}\right)$ defined inductively. Let $f_{0}=f$. Now suppose inductively that $f_{i}$ is the path

$$
\begin{aligned}
& x_{k} \frac{a_{k}}{a_{k}} \\
& \vdots \\
& \begin{array}{r}
x_{i+1} \\
x_{i} \frac{}{a_{i}} y_{i}
\end{array} \\
& x_{i} \frac{c_{i}}{c_{i}} x_{i} \quad \cdots \\
& x_{i} \frac{c_{i}}{c_{i}} x_{i} \\
& \begin{array}{ccccccccc}
c_{i} & c_{i} & c_{i} & \ldots & c_{i} & c_{i+1} & \ldots & c_{k} & c_{k+1}
\end{array}
\end{aligned}
$$

Then we can take a horizontal step, obtaining $p_{i}$, which is

$$
\begin{aligned}
& x_{k} \frac{a_{k}}{a_{k}} \\
& \begin{array}{r}
x_{i+1} \\
y_{i} \frac{}{c_{i+1}} y_{i}
\end{array} \\
& y_{i} \frac{c_{i+1}}{c_{i}} \quad \cdots \\
& y_{i} \frac{c_{i+1}}{c_{i}} \\
& \begin{array}{lllllllll}
c_{i+1} & c_{i+1} & c_{i+1} & \ldots & c_{i+1} & c_{i+1} & \ldots & c_{k} & c_{k+1}
\end{array}
\end{aligned}
$$

We have $\left\langle a_{i}, \ldots, a_{i}, c_{i+1}, \ldots, c_{k+1}\right\rangle \in\left[f_{i} \sim p_{i}\right]$. Subsequently, we take a vertical step, obtaining $f_{i+1}$ from $p_{i}$. Clearly, $p_{k}$ is the constant path at $y_{k}$. This defines the function $\ell_{k}$.

In order to see that $\ell_{k}$ really induces a morphism $(X, \sim)^{I_{k}} \rightarrow\left((X, \sim)^{I_{k}}\right)^{I_{k}}$, suppose $f, g$ represent elements of $(X, \sim)^{I_{k}}$ and $\gamma=\left\langle\gamma_{0}, \ldots, \gamma_{k+1}\right\rangle \in[f \sim g]$. If $f$ is the sequence $\left(x_{0}, y_{0}, \ldots, x_{k}, y_{k}\right)$ and $g$ is $\left(z_{0}, w_{0}, \ldots, z_{k}, w_{k}\right)$, then $\gamma_{0} \in\left[x_{0} \sim z_{0}\right], \gamma_{i} \in\left[x_{i} \sim z_{i}\right] \cap\left[y_{i-1} \sim w_{i-1}\right]$ for $1 \leqslant i \leqslant k$, and $\gamma_{k+1} \in\left[y_{k} \sim w_{k}\right]$.

Then if $\ell_{k}(f)=\left(f_{0}, p_{0}, \ldots, f_{k}, p_{k}\right)$ and $\ell_{k}(g)=\left(g_{0}, h_{0}, \ldots, g_{k}, h_{k}\right)$, one sees by induction that $\gamma \in\left[f_{0} \sim g_{0}\right]$, that $\left\langle\gamma_{i}, \ldots, \gamma_{i}, \gamma_{i+1}, \ldots, \gamma_{k+1}\right\rangle$ is an element of $\left[f_{i} \sim g_{i}\right] \cap\left[p_{i-1} \sim h_{i-1}\right]$ for $1 \leqslant i \leqslant k$, and $\left\langle\gamma_{k+1}, \ldots, \gamma_{k+1}\right\rangle$ is in $\left[p_{k} \sim h_{k}\right]$. So if $\rho_{i}=\left\langle\gamma_{i}, \ldots, \gamma_{i}, \gamma_{i+1}, \ldots, \gamma_{k+1}\right\rangle$, then

$$
\rho=\left\langle\rho_{0}, \ldots, \rho_{k+1}\right\rangle \in\left[\ell_{k}(f) \sim \ell_{k}(g)\right] .
$$

So we have a well-defined morphism $L_{k}:(X, \sim)^{I_{k}} \rightarrow\left((X, \sim)^{I_{k}}\right)^{I_{k}}$.

The next step is to see that the maps $L_{k}$ extend to a map $L: \mathrm{P}(X, \sim) \rightarrow \operatorname{PP}(X, \sim)$ : that is, that they interact well with the equality relation on $\mathrm{P}(X, \sim)$. 
Let $\ell$ be the function which sends $(k, f)$ (where $f$ represents an element of $(X, \sim)^{I_{k}}$ ) to $\left(k, \ell_{k}(f)\right)$. We have to see that there is a computable function $\phi$ such that whenever $a \in[(k, f) \sim(m, g)]$ then $\phi(a) \in[\ell(k, f) \sim \ell(m, g)]$. From $a$ we can computably extract $k$ and $m$ and hence their maximum; say this is $m$. Then we only have to consider order and endpoint preserving maps $I_{m} \rightarrow I_{k}$. Such a map is a composition of maps $\sigma_{j}$ induced by functions $t_{j}:\{0, \ldots, k+1\} \rightarrow\{0, \ldots, k\}$ of the form: $t_{j}(i)=i$ for $i \leqslant j$, and $t_{j}(i)=i-1$ otherwise. Then the resulting map $(X, \sim)^{\sigma_{j}}:(X, \sim)^{I_{k}} \rightarrow(X, \sim)^{I_{k+1}}$ is induced by the function $s_{j}$ which sends

$$
f=\left(x_{0}, y_{0}, \ldots, x_{k}, y_{k}\right)
$$

to

$$
s_{j}(f)=\left(x_{0}, y_{0}, \ldots, x_{j}, x_{j}, x_{j}, y_{j}, x_{j+1}, \ldots, x_{k}, y_{k}\right) .
$$

Suppose, $a \in[(k, f) \sim(k+1, g)]$ so (ignoring irrelevant information) $a \in\left[s_{j}(f) \sim g\right]$ in $(X, \sim)^{I_{k+1}}$. From $a$ we find elements $a_{1} \in E\left(s_{j}(f)\right), a_{2} \in E(f)$ and $a_{3} \in\left[\ell_{k+1}\left(s_{j}(f)\right) \sim\right.$ $\ell_{k+1}(g)$ ] (the last since $\ell_{k+1}$ induces a morphism as we have seen). It suffices therefore to find an element of

$$
\left[\left(k, \ell_{k}(f)\right) \sim \ell_{k+1}\left(s_{j}(f)\right)\right]
$$

which does not depend on $j$.

Now from the definition of $\ell_{k}$ it is clear that if $\ell_{k}(f)=\left(f_{0}, g_{0}, \ldots, f_{k}, g_{k}\right)$ then

$$
\begin{aligned}
\ell_{k+1}\left(s_{j}(f)\right) & =\left(s_{j}\left(f_{0}\right), s_{j}\left(g_{0}\right), \ldots, s_{j}\left(f_{j}\right), s_{j}\left(g_{j}\right), s_{j}\left(f_{j}\right), s_{j}\left(g_{j}\right), s_{j}\left(f_{j+1}\right), \ldots, s_{j}\left(g_{k}\right)\right) \\
& =s_{j}\left(s_{j}\left(f_{0}\right), s_{j}\left(g_{0}\right), \ldots, s_{j}\left(f_{k}\right), s_{j}\left(g_{k}\right)\right) .
\end{aligned}
$$

So from $a_{3}$ we find an element of

$$
\left[\ell_{k+1}\left(s_{j}(f)\right) \sim\left(s_{j}\left(f_{0}\right), \ldots, s_{j}\left(g_{k}\right)\right)\right]
$$

and successively elements of $\left[s_{j}\left(f_{i}\right) \sim f_{i}\right]$, hence (by the equality of function spaces) an element of

$$
\left[\left(s_{j}\left(f_{0}\right), \ldots, s_{j}\left(g_{k}\right)\right) \sim\left(f_{0}, \ldots, g_{k}\right)\right] .
$$

Combining, we get the desired element of $\left[\ell_{k+1}\left(s_{j}(f)\right) \sim \ell_{k}(f)\right]$.

\section{Discrete and path connected objects}

In the effective topos, an object is called discrete if it is a subquotient of the object of natural numbers $N$. The object $N$ is the canonically projective object with underlying set $\mathbb{N}$, and $E(n)=\{n\}$. The following characterization of the discrete objects is taken from Van Oosten (2008), 3.2.20.

Proposition 3.1. An object $(X, \sim)$ of $\mathcal{E} f f$ is discrete if and only if there is a computable function $\phi$ such that for all $x, x^{\prime} \in X$ we have: if $n \in E(x) \cap E\left(x^{\prime}\right)$ then $\phi(n) \in\left[x \sim x^{\prime}\right]$. This is the case if and only if $(X, \sim)$ is isomorphic to an object $(Y, \sim)$ for which we have $E(y) \cap E\left(y^{\prime}\right)=\varnothing$ whenever $y \neq y^{\prime}$.

Let $\mathcal{E} f f_{d}$ be the full subcategory of $\mathcal{E} f f$ on the discrete objects. $\mathcal{E} f f_{d}$ is a very rich category: it contains all of 'constructive mathematics'. 
The inclusion functor $\mathcal{E} f f_{d} \rightarrow \mathcal{E f f}$ has a left adjoint which preserves products: the discrete reflection.

Definition 3.2 (Van Oosten (2008), 3.2.19iii)). The discrete reflection of an object $(X, \sim)$, denoted $(X, \sim)_{d}$, is the object with underlying set $\bigcup_{x \in X} E(x)$, and with the following equality: $[n \sim m]$ consists of all coded sequences

$$
\sigma=\left\langle n_{0}, a_{0}, n_{1}, \ldots, n_{k}, a_{k}, n_{k+1}\right\rangle
$$

satisfying: for each $i \leqslant k$ there are $x_{i}, y_{i} \in X$ such that $n_{i} \in E\left(x_{i}\right), n_{i+1} \in E\left(y_{i}\right)$ and $a_{i} \in\left[x_{i} \sim y_{i}\right]$.

The universal arrow $\eta:(X, \sim) \rightarrow(X, \sim)_{d}$ is represented by the function

$$
H(x, m)=\bigcup_{n \in E(x)}[n \sim m] .
$$

It is easily verified that $\eta$ is always an epimorphism. Now from the definition of $\mathrm{P}(X, \sim)$ in the previous section it should be obvious that the kernel pair of $\eta$ is just the image of the map $(\mathrm{s}, \mathrm{t}): \mathrm{P}(X, \sim) \rightarrow(X, \sim) \times(X, \sim)$. Furthermore, since $\mathcal{E} f f_{d}$ is a full subcategory of $\mathcal{E} f f$ we have that $\eta$ is an isomorphism if and only if $(X, \sim)$ is discrete. Summarizing:

\section{Proposition 3.3.}

i. An object $(X, \sim)$ of $\mathcal{E} f f$ is discrete if and only if it is internally true that there are no nonconstant paths (i.e. the map $\mathrm{c}:(X, \sim) \rightarrow \mathrm{P}(X, \sim)$ is an isomorphism).

ii. The discrete reflection is, internally, the set of path components.

We shall therefore call an object path connected if its discrete reflection is isomorphic to 1 , the terminal object of $\mathcal{E f f}$.

\section{Homotopy and fundamental group(oid)}

Two maps $f, g:(X, \sim) \rightarrow(Y, \sim)$ are homotopic if there exists a homotopy from $f$ to $g$, that is: a map $H:(X, \sim) \rightarrow \mathrm{P}(Y, \sim)$ such that $\mathrm{s} H=f$ and $\mathrm{t} H=g$. By composition of paths, converse paths and constant paths, homotopy is an equivalence relation.

For paths in $(X, \sim)$ there is the further notion of endpoint preserving homotopy. This is an element $H$ of $\mathrm{PP}(X, \sim)$ such that both $\mathrm{P}(\mathrm{s})(H)$ and $\mathrm{P}(\mathrm{t})(H)$ are constant paths. The picture is:

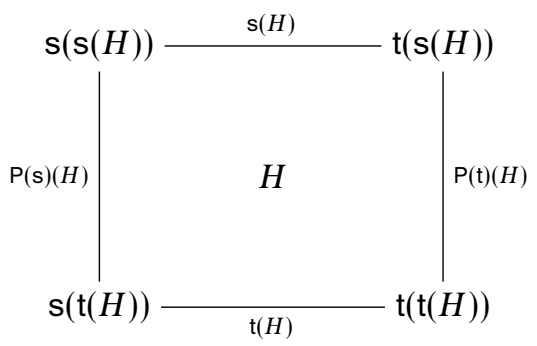


Composition of homotopies is 'vertical composition':

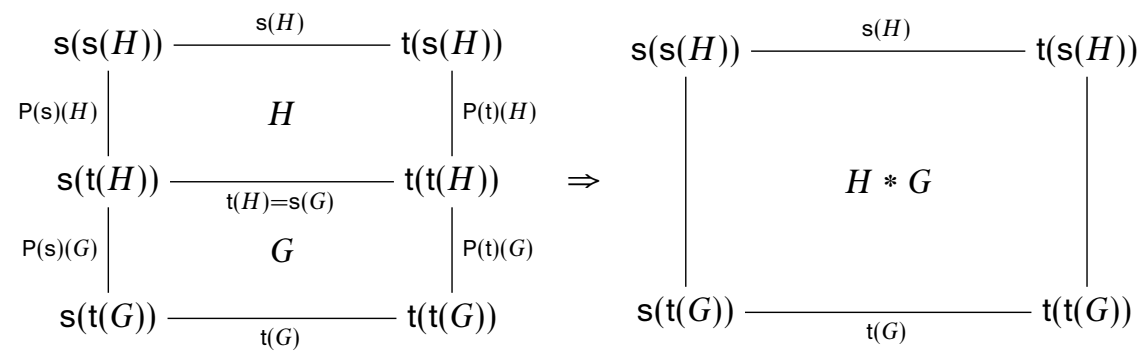

We also have 'horizontal composition': if $\mathrm{P}(\mathrm{t})(H)=\mathrm{P}(\mathrm{s})(G)$ then the pair $(H, G)$ is actually a path in the object $\mathrm{P}(X, \sim) \times_{(X, \sim)} \mathrm{P}(X, \sim)$ and we have $H \circ G=\mathrm{P}(*)(H, G)$ :

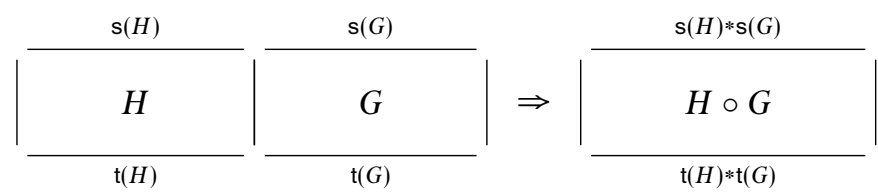

which is a homotopy from $\mathrm{s}(H) * \mathrm{~s}(G)$ to $\mathrm{t}(H) * \mathrm{t}(G)$.

It is easy to see that the map $L$ from Proposition 2.7, satisfying $s L(f)=f$ and $\mathrm{t} L(f)=\mathrm{ct}(f)$, preserves the endpoint: $\mathrm{P}(\mathrm{t})(L(f))=\mathrm{c}(\mathrm{t}(f))$.

Using this map $L$ and horizontal composition, one readily verifies that there is, for each $f \in \mathrm{P}(X, \sim)$, and endpoint preserving homotopy from $\mathrm{c}(\mathrm{s}(f))$ to $f * \tilde{f}$.

Definition 4.1. Let $(X, \sim)$ be an object of $\mathcal{E f f}$ and $x: 1 \rightarrow(X, \sim)$ a base point. The fundamental group of $(X, \sim)$ with base point $x$, or $\pi_{1}((X, \sim), x)$, is the set of endpoint preserving homotopy classes of paths from $x$ to $x$, with composition of paths as operation.

The fundamental group is in fact a group: composition is well defined on homotopy classes by horizontal composition, and strictly associative, the constant path on $x$ is the unit, and $\tilde{f}$ is a two-sided inverse for $f$. As usual, if $(X, \sim)$ is path connected the fundamental group does not really depend on the base point and we can omit it.

Definition 4.2. An object is simply connected if it is path connected and its fundamental group is trivial.

We can also define, for each $(X, \sim)$, the fundamental groupoid of $(X, \sim)$ : its object of objects is $(X, \sim)$, its object of arrows is the object of endpoint preserving homotopy classes of paths.

As an example, I now briefly discuss 'circles'. A circle, naturally, is constructed by identifying the endpoints of an interval.

Definition 4.3. The circle $C_{n}$ is defined by the coequalizer diagram

$$
1 \stackrel{0}{\underset{n}{\longrightarrow}} \tilde{I}_{n} \longrightarrow C_{n}
$$


Proposition 4.4. For $n \neq 2$, the object $C_{n}$ is separated. In fact, $C_{0} \cong C_{1} \cong 1$ and for $n>2$, the object $C_{n}$ is isomorphic to the assembly $(\{0, \ldots, n-1\}, E)$ with

$$
E(0)=\{0,1\}, \ldots, E(n-2)=\{n-2, n-1\}, E(n-1)=\{n-1,0\} .
$$

Proof. Straightforward calculation.

One can show that the object $C_{2}$, which has 2 points, is not separated.

Proposition 4.5. For each $n>2, \pi_{1}\left(C_{n}\right) \cong \mathbb{Z}$.

Proof. This follows standard lines. First one defines the 'line' $R$ : it is the object $(\mathbb{Z}, E)$ with

$$
\begin{aligned}
& E(n)=\{2 n-1,2 n+1\} n>0 \\
& E(0)=\{0,1\} \\
& E(n)=\{-2 n,-2 n-2\} n<0
\end{aligned}
$$

One observes that $R$ is simply connected and that the map $R \rightarrow C_{n}$ given by $m \mapsto m \bmod n$ is a 'universal covering': it has the unique path lifting and homotopy lifting properties.

Consider both $R$ and $C_{n}$ as equipped with the base point 0 . The map from $\pi_{1}\left(C_{n}, 0\right)$ to $R$, sending a homotopy class of a path to the target of its lifting, is well-defined and gives a bijection from $\pi_{1}\left(C_{n}, 0\right)$ to $\mathbb{Z}$. Composition of paths corresponds under this map to addition, so the required isomorphism is there.

Of course, in $\mathcal{E} f f$ we also have 'tori' $T_{n}=C_{n} \times C_{n}$ and since we can prove that $\pi_{1}$ is a functor from $\mathcal{E} f f_{*}$ (the category of objects of $\mathcal{E} f f$ with a base point, and base point preserving maps) to the category of groups and preserves products, we have

$$
\pi_{1}\left(T_{n}\right)=\mathbb{Z} \times \mathbb{Z}
$$

for $n>2$.

Similarly, we can discuss (at least finite) 'wedges of circles'. 'Spheres' would be quotients of cubes (products of intervals).

\section{Homotopy equivalences and Hurewicz fibrations}

\section{Definition 5.1.}

a. An arrow $f:(X, \sim) \rightarrow(Y, \sim)$ embeds $(X, \sim)$ as strong deformation retract into $(Y, \sim)$ if there is a map $F:(Y, \sim) \rightarrow \mathrm{P}(Y, \sim)$ with the properties:

i. $\mathrm{s} F=\operatorname{id}_{(Y, \sim)}$,

ii. the diagram

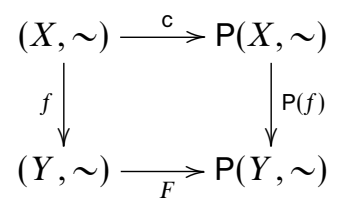

commutes, i.e. for $x \in(X, \sim), F(f(x))$ is the constant path on $f(x)$,

iii. the map $\mathrm{t} F$ factors through $f$. 
b. An arrow $f:(X, \sim) \rightarrow(Y, \sim)$ is a homotopy equivalence if there is an arrow $g:(Y, \sim)$ $\rightarrow(X, \sim)$ such that $\mathrm{fg}$ is homotopic to $\operatorname{id}_{(Y, \sim)}$ and $g f$ is homotopic to $\operatorname{id}_{(X, \sim)}$.

Clearly, every embedding as strong deformation retract is a homotopy equivalence.

Proposition 5.2. The map $\mathrm{c}:(X, \sim) \rightarrow \mathrm{P}(X, \sim)$ embeds $(X, \sim)$ as strong deformation retract into $\mathrm{P}(X, \sim)$.

Proof. Use $L$.

Definition 5.3. Given $f:(X, \sim) \rightarrow(Y, \sim)$, denote by $C_{f}$ the object defined by the pullback square

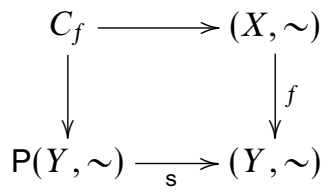

So, $C_{f}=\{(\alpha, x) \in \mathrm{P}(Y, \sim) \times(X, \sim) \mid \mathrm{s}(\alpha)=f(x)\}$.

Let $v_{f}: \mathrm{P}(X, \sim) \rightarrow C_{f}$ be the map defined by the commutative diagram

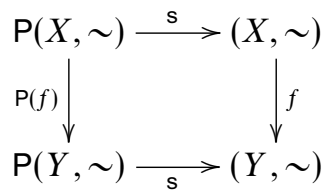

i.e. $v_{f}(\omega)=(\mathrm{P}(f)(\omega), \mathrm{s}(\omega))$.

The map $f$ is called a Hurewicz fibration if the map $v_{f}$ has a section.

Our definition of 'Hurewicz fibration' is equivalent to the standard one for topological spaces (this is usually considered a theorem: a map is a Hurewicz fibration if and only if it has a Hurewicz connection), and is a rewrite in a language with no interval, but only path objects.

Proposition 5.4. Every arrow $f:(X, \sim) \rightarrow(Y, \sim)$ in $\mathcal{E} f f$ factors as an embedding as strong deformation retract, followed by a Hurewicz fibration.

Proof. This follows the usual definition for topological spaces. Factor $f$ as

$$
(X, \sim) \stackrel{l}{\longrightarrow} C_{f} \stackrel{\pi}{\longrightarrow}(Y, \sim)
$$

where $l(x)=(x, \mathrm{c}(f(x)))$ and $\pi(x, \omega)=\mathrm{t}(\omega)$. Analogously to the embedding $(X, \sim) \rightarrow$ $\mathrm{P}(X, \sim), l$ is easily seen to be an embedding as strong deformation retract. For the other map $\pi$, we have to show that the map

$$
\mathrm{P}\left(C_{f}\right) \stackrel{(\mathrm{s}, \mathrm{P}(\pi))}{\rightarrow} C_{\pi}
$$

has a section.

The object $C_{\pi}$ is internally defined as

$$
C_{\pi}=\left\{((x, \omega), \eta) \in C_{f} \times \mathrm{P}(Y, \sim) \mid f(x)=\mathrm{s}(\omega), \mathrm{t}(\omega)=\mathrm{s}(\eta)\right\} .
$$


A typical element of $\mathrm{P}\left(C_{f}\right)$ is a pair $(\alpha, H)$ where $\alpha$ is a path in $(X, \sim)$ and $H$ is a path in $\mathrm{P}(Y, \sim)$ with $\mathrm{P}(\mathrm{s})(H)=\mathrm{P}(f)(\alpha)$, so $f(\mathrm{~s}(\alpha))=\mathrm{s}(\mathrm{s}(H))$.

Now for $((x, \omega), \eta) \in C_{\pi}$ we have to find $(\alpha, H)$ such that $\mathrm{s}(H)=\omega$ and $\mathrm{P}(\mathrm{t})(H)=\eta$. But we can use Proposition 2.7 again: let $\alpha$ be $c(f(x))$ and $H$ be the path from $\omega$ to $\omega * \eta$ such that $\mathrm{P}(\mathrm{s})(H)$ is constant on $f(x)$ and $\mathrm{P}(\mathrm{t})(H)=\eta$. So indeed, $\pi$ is a Hurewicz fibration. $\square$

\section{Remarks}

1. In an old paper by Strøm (1972) it is shown that there is a closed model structure on the category of topological spaces in which the fibrations are the Hurewicz fibrations, the weak equivalences are the homotopy equivalences and the cofibrations are the 'Hurewicz cofibrations', that is in our terminology: all maps which have the left lifting property with respect to all maps $\mathrm{P}(X) \stackrel{\mathrm{s}}{\rightarrow} X$. I have not been able to find good factorizations as Hurewicz cofibrations followed by trivial fibrations, and I doubt they exist.

2. Baues (1989) proposes the following relaxation of the notion of closed model structure: the notion of a fibration category. It is a category together with two classes of maps, the fibrations and the weak equivalences satisfying the following requirements (in stating them, I assume that we work in a category with pullbacks):

F1. Every isomorphism is both a fibration and a weak equivalence; fibrations are closed under composition and weak equivalences satisfy the 2-out-of-3 property.

F2. Fibrations are stable under pullback and weak equivalences are stable under pullback along fibrations. Also, trivial fibrations (that is: fibrations which are also weak equivalences) are stable under pullback.

F3. Every arrow factors as a weak equivalence followed by a fibration.

F4. Call an object cofibrant if every trivial fibration into it has a section. For each object $X$ there is a cofibrant object $Q X$ and a trivial fibration $Q X \rightarrow X$.

The structure we described on $\mathcal{E} f f$ with homotopy equivalences as weak equivalences, and Hurewicz fibrations as fibrations, satisfies at least F1, F3 and F4. That F4 holds is an easy consequence of the observation that every Hurewicz fibration which is also a homotopy equivalence, must have a section, so every object is cofibrant. As to F2, it is straightforward from the fact that the functor $\mathrm{P}$ preserves pullbacks, that Hurewicz fibrations are stable under pullback. That homotopy equivalences are stable under pullback along Hurewicz fibrations is something I strongly feel should be true (the Strøm model structure is 'right proper'), but have not been able to prove.

3. In Van den Berg and Garner (2012), Van den Berg and Garner develop the notion of a path object category. It is a matter of reading through the axioms to see, that they are satisfied in our homotopy interpretation in $\mathcal{E} f f$. Therefore, by that paper, our results imply that there should be a model for the intensional identity types in Martin-Löf type theory.

\section{Acknowledgements}

I like to thank Benno van den Berg and Ieke Moerdijk for fruitful discussions. 
Basic research for this paper was done while the author spent the Fall of 2009 at the Mittag-Leffler Institute in Stockholm, in the program Set theory and Model theory. $\mathrm{He}$ is deeply grateful to both the organizers of this programme (for having invited him, although he is neither a set theorist nor a model theorist) and to the dedicated staff of the MLI (for making his stay pleasant as well as fruitful).

\section{References}

Awodey, S. and Warren, M. (2009) Homotopy theoretic models of identity types. Mathematical Proceedings of the Cambridge Philosophical Society 146 (1) 45-55.

Baues, H. J. (1989) Algebraic homotopy, Cambridge studies in advanced mathematics volume 15, Cambridge University Press.

Dwyer, W. G. and Spalinski, J. (1995) Homotopy theories and model categories. In: James, I. M. (ed.) Handbook of Algebraic Topology, Elsevier.

Gambino, N. and Garner, R. (2008) The identity weak factorization system. Theoretical Computer Science 409 (1) 94-109.

Hirschhorn, P. S. (2003) Model Categories and Their Localizations, Mathematical Surveys and Monographs volume 99, AMS.

Hovey, M. (1999) Model Categories, Mathematical Surveys and Monographs volume 63, AMS.

Hyland, J. M. E. (1982) The effective topos. In: Troelstra, A. S. and Van Dalen, D. (eds.) The L.E.J. Brouwer Centenary Symposium, North Holland Publishing Company 165-216.

Hyland, J. M. E., Robinson, E. P. and Rosolini, G. (1990) The discrete objects in the effective topos. Proceedings of the London Mathematical Society $601-60$.

Kapulkin C., Lumsdaine, P. L. and Voevodsky, V. (2012) The simplicial model of univalent foundations. Available at: arXiv:1211.2851v1.

Quillen, D. G. (1967) Homotopical Algebra, Lecture Notes in Mathematics volume 43, Springer.

Strøm, A. (1972) The homotopy category is a homotopy category. Archiv der Mathematik XXIII 435-441.

The Univalent Foundations Program. (2013) Homotopy Type Theory, Institute for Advanced Study, Princeton. Collectively written account of HoTT by participants of the Special Year on Univalent Foundations of Mathematics, organized by S. Awodey, Th. Coquand and V. Voevodsky.

van den Berg, B. and Garner, R. (2011) Types are weak $\omega$-groupoids. Proceedings of the London Mathematical Society 102 (3) 370-394.

van den Berg, B. and Garner, R. (2012) Topological and simplicial models of identity types. ACM Transactions on Computational Logic (TOCL) 13(1) 1-44.

van Oosten, J. (2008) Realizability: An Introduction to its Categorical Side, Studies in Logic volume 152, North-Holland.

Voevodsky, V. (2010) The equivalence axiom and univalent models of type theory. Talk at CMU.

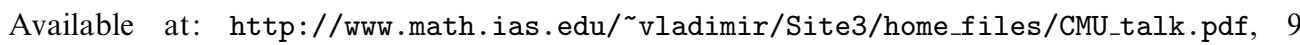
pages.

Warren, M. (2008) Homotopy theoretic Aspects of Constructive Type Theory, Ph.D. thesis, Carnegie Mellon University. 